\title{
Doenças e agravos de notificação compulsória e condições socioambientais: estudo ecológico, Espírito Santo, 2011-2015*
}

doi: 10.1590/S1679-49742021000200019

\section{Compulsorily notifiable diseases and health problems and socio-environmental conditions: an ecological study, Espírito Santo, Brazil, 2011-2015}

\author{
Enfermedades y trastornos de notificación obligatoria y condiciones socioambientales: \\ estudio ecológico, Espírito Santo, Brasil, 2011-2015
}

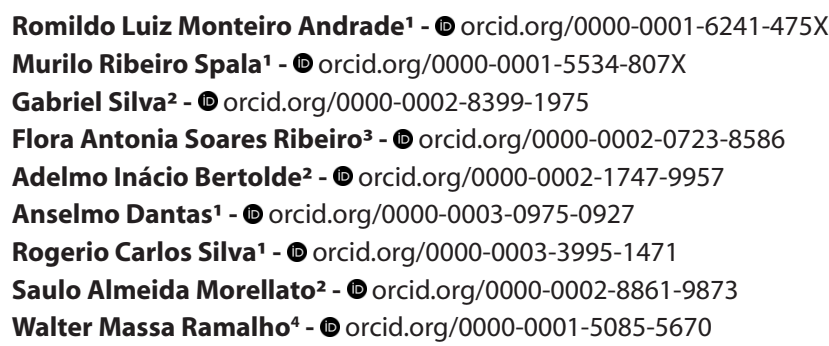

\section{Resumo}

Objetivo: Analisar a associação das condições climático-ambientais e a ocorrência das doenças e agravos de notificação compulsória no estado do Espírito Santo, Brasil. Métodos: Estudo ecológico dos agregados municipais calculados a partir de casos confirmados no Sistema de Informação de Agravos de Notificação (Sinan) para o período 2011-2015. Resultados: As notificações foram mais frequentes no sexo feminino (51,1\%), raça/cor da pele parda (31,7\%), faixa etária de 20-49 anos $(48,1 \%)$ e região metropolitana de saúde $(60,3 \%)$. Os fatores associados aos agravos foram as condições sensíveis à Atenção Básica (p-valor $<0,001)$, o índice de desenvolvimento de educação ( $\mathrm{p}$-valor $<0,001)$, a temperatura ( -valor=0,019) e 0 grau de urbanização ( $\mathrm{p}$-valor $=0,004)$. As doenças estiveram associadas a densidade populacional ( $\mathrm{p}$-valor $<0,001)$, temperatura ( $\mathrm{p}$-valor $<0,001$ ), umidade ( $\mathrm{p}$-valor $<0,001$ ) e altitude ( $\mathrm{p}$-valor=0,005). Conclusão: Os agravos associaram-se positivamente às condições sensíveis à Atenção Básica, índice de desenvolvimento da educação e temperatura; e negativamente ao grau de urbanização. As doenças associaram-se positivamente aos fatores citados.

Palavras-chave: Incidência; Notificação de Doenças; Fatores Socioeconômicos; Análise Espacial; Meio Ambiente.

*A pesquisa recebeu apoio da Secretaria de Estado da Saúde do Espírito Santo, gerenciado pela Fundação de Amparo à Pesquisa e Inovação do Espírito Santo (Fapes/ES).

Endereço para correspondência:

Adelmo Inácio Bertolde - Universidade Federal do Espírito Santo, Departamento de Estatística, Av. Fernando Ferrari, no 514 Goiabeiras, Vitória, ES, Brasil. CEP: 29075-910

E-mail: adelmo.bertolde@ufes.br 


\section{Introdução}

A compreensão da relação saúde-ambiente encontra-se em destaque, especialmente com as transformações climáticas em curso e seu impacto sobre 0 adoecimento populacional. Os registros das influências ambientais na distribuição do processo saúde-doença datam do século $\mathrm{V}$ a.C., descritos por Hipócrates. ${ }^{1}$ Nos dias de hoje, o Painel Intergovernamental sobre Mudanças Climáticas trouxe à tona reflexões de reconsideração dos impactos das condições ambientais na ocorrência de doenças, sugerindo que os efeitos deletérios das mudanças climáticas sobre 0 planeta devam ser mitigados em nome da proteção e promoção da saúde humana, mediante a nominada 'saúde ambiental'., ${ }^{2,3}$ Nesse sentido, a compreensão ampliada da inter-relação do binômio 'ambiente e saúde' ressignifica o lugar como condicionante do adoecimento, estimulando análises dos impactos das mudanças socioambientais e suas consequências para o cenário sanitário, local e global. ${ }^{4,5}$

\section{A compreensão ampliada da interrelação do binômio 'ambiente e saúde' ressignifica o lugar como condicionante do adoecimento.}

Estudos nacionais que abordam a incidência das doenças e agravos de notificação compulsória (DANC) empregam técnicas de distribuição espacial com 0 propósito de identificar áreas de risco, e fornecer subsídios para a priorização de recursos financeiros visando seu enfrentamento. ${ }^{6-8}$ No estado do Espírito Santo, estudos de distribuição espacial abordam recortes específicos de doenças ou agravos, embora sob uma abordagem individualizada., ${ }^{9,10}$

Distintamente, este trabalho busca uma abordagem holística, considerando as DANC e suas vias de transmissão comuns. É o caso das arboviroses, zoonoses, infecções sexualmente transmissíveis ou características comuns à sua ocorrência, como é o caso das doenças ocupacionais ou, ainda, do modo de prevenção comum para o caso das doenças imunopreveníveis.

O Sistema Nacional de Vigilância Epidemiológica teve sua criação oficializada em 1975, após recomendação da $8^{a}$ Conferência Nacional de Saúde, estabelecendo as competências para identificação e registro dos eventos de notificação compulsória no âmbito da Saúde Pública. ${ }^{11}$ Considera-se como 'evento de notificação' todo caso que

\begin{abstract}
"apresente risco de propagação ou disseminação de doenças para mais de uma unidade federada, com prioridade para doenças de notificação imediata e outros eventos de saúde pública, independentemente da natureza ou origem, que possa necessitar de resposta nacional imediata." ${ }^{12}$
\end{abstract}

A Lista Nacional de Notificação Compulsória é atualizada periodicamente. No momento desta publicação, encontra-se em vigor a Portaria de Consolidação $\mathrm{n}^{0}$ 4, datada de 28 de setembro de 2017, Anexo V da Portaria MS/GM 204, de 17 de fevereiro de 2016, atualizada pela Portaria GM/MS n ${ }^{\circ} 1061$, de 18 de maio de 2020. Os registros de doenças e agravos de notificação compulsória são feitos no Sistema de Informação de Agravos de Notificação (Sinan). ${ }^{13} \mathrm{~A}$ primeira versão do Sinan, de 1993, foi elaborada no formato Disk Operating System-DOS, atualizado para Sinan-Windows em 1998 e pela versão online it. Sinan-NET de 2006. O Sinan é alimentado pelas unidades municipais, com o preenchimento de dados no nível local, na Ficha Individual de Notificação (FIN). Esta ficha dispõe de campos destinados aos registros das coordenadas geográficas de latitude (ID_GE01) e longitude (ID_GEO2); entretanto, sua extração não se encontra disponível nos sistemas de tabulação online (TABNET).

A abordagem do estudo em tela pretende demonstrar os efeitos das condições de iniquidade em saúde associadas às variáveis climático-ambientais e à ocorrência das DANC, mediante o emprego de técnicas de geoprocessamento, com propósito de investigar possíveis condições associadas, segundo 0 modelo ecossocial envolvido na determinação de sua ocorrência. ${ }^{14}$ Este relato da investigação desenvolvida teve como objetivo aferir a relação entre as iniquidades sociais, (i) as condições climático-ambientais e (ii) a ocorrência das DANC no Espírito Santo, no período de 2011 a 2015.

\section{Métodos}

Trata-se de um estudo ecológico longitudinal, por agregados municipais, segundo critérios de definição dos casos confirmados de DANC em residentes no estado do Espírito Santo, no período de 2011 a 2015. 
Os casos participantes foram extraídos do Sinan e tabulados em 29 de maio de 2019, em arquivo padrão Nindinet.dbf, disponibilizado pelo Núcleo Especial de Sistemas de Informação em Saúde da Gerência de Vigilância em Saúde, da Secretaria de Estado da Saúde do Espírito Santo (NESIS/GEVS/SESA). A extração inicial contou com 511.912 registros, submetidos aos filtros dos campos <número de notificação $>$, < nome do notificado $>,<$ nome da mãe $>,<$ data de notificação $>$ e $<$ nome do agravo $>$ e local de residência, para identificação das duplicidades ( 54.449 casos), perfazendo o total de 347.789 casos - asseguradas a completitude e a consistência dos registros.

O Espírito Santo está situado no litoral do Sudeste brasileiro, faz divisa com os estados da Bahia ao norte, Minas Gerais a oeste e noroeste, e Rio de Janeiro ao sul. Os capixabas (como são mais conhecidos os espírito-santenses) dispõem de uma área territorial de $46.095,583 \mathrm{~km}^{2}$, apresentam uma densidade populacional de 76,25 habitantes $/ \mathrm{km}^{2}$ e habitam 78 municípios, distribuídos entre as quatro regiões de saúde do estado. ${ }^{20,21}$

Os casos confirmados foram incluídos e agrupados segundo características etiológicas comuns, uma vez identificados pela Décima Classificação Internacional de Doenças e Problemas Relacionados à Saúde (CID-10):

a) Arboviroses: A90; A92.0; A92.8; A95.9;

b) Doenças imunopreveníveis: A35; A36.9; A37.9; A80.9; D01.9; B019; G03.9; J09; J11; J18.9; P35.0; Y59; B19 (hepatite B);

c) Causas externas: T65.9; X29; Y09; Y96; Z20.9

d) Doenças negligenciadas: A19.6; A30.9; B54; B55.0; B55.1; B57.1; B65.9;

e) Infecções sexualmente transmissíveis (IST): A50.9; A53.9; B24; B19 (hepatite C); B54; 098.1; R36; Z20.6; Z21;

f) Zoonoses: A23; A27.9; A77.9; A82.9; A98.8; B58; 098.6; P36.1; W64;

g) Doenças transmitidas por água e alimentos (DTAA): A00.9; A01.0; A05.1; A08.0; B19 (hepatite A)

h) Doenças ocupacionais: C80; F99; H83.3; J64; L98.9; Z57.9;

i) Hepatites sem confirmação etiológica; e

j) Grupo residual: A05.9; A22.9; A69.2; A81.0; R17. A execução do trabalho cumpriu as seguintes etapas:

1) Obtenção dos dados em arquivos extraídos do Sinan/ES, posteriormente analisados com uso do software Stata versão $16 \mathrm{MP}$.
2) Análise crítica do banco de dados, com a finalidade de excluir duplicidades e inconsistências.

3) Extração das coordenadas geográficas, a partir do endereço das fichas de notificação, com auxílio e desenvolvimento de ferramentas automatizadas de consultas online. A validação do endereço utilizou oito combinações de endereçamento, sendo a combinação 'endereço completo' composta pelos seguintes dados: logradouro; número; bairro; município; estado; e país.

4) Análise exploratória de dados

Foram obtidas estatísticas sumárias das variáveis biológicas (sexo [feminino; masculino]; raça/cor da pele [amarela; branca; indígena; parda; preta], faixa etária [em anos: menor que 1; 0 a 4; 5 a 9; 10 a 19; 20 a 49; 50 a 79; 80 ou mais], variáveis geográficas (regiões de saúde); e variáveis etiológicas (CID-10).

5) Em seguida, aplicou-se a técnica de análise fatorial (AF), tomando-se as taxas de incidências das 30 doenças e agravos com maior número de casos (perfazendo 347 mil casos, 99,8\% do total), com a finalidade de identificar padrões de distribuição espacial.

6) Modelagem socioambiental

Foram considerados os modelos de regressão de Poisson e regressão binomial negativa, aplicados aos agravos (códigos: W64; X29; T659; Y09; Z209; Y96; Z579 e Y59) e doenças (demais codificações). Nos ajustes para escolha do modelo, utilizaram-se os critérios de informação de Akaike e a significância estatística do parâmetro de dispersão da regressão binomial negativa. Os resultados dos modelos de regressão destacam as relações significantes, expressas em: $\beta ;$ p-valor. Para a avaliação do efeito longitudinal nos cinco anos do estudo, verificou-se a presença de correlação temporal, com emprego da abordagem marginal por cópulas gaussianas. ${ }^{16}$

7) Análise multivariada

Empregou-se a técnica de análise fatorial (AF) exploratória, a partir das taxas de incidência municipais, para identificar fatores comuns e sua relação com a carga das DANC. As estimativas para os modelos de análise fatorial e de regressão foram obtidas utilizando-se o software R, considerando-se o nível de significância de $10 \%$ para os testes de hipóteses.

0 constructo relacional utilizado contempla três níveis hierárquicos de exposição associados ao desfecho da incidência das DANC, que referenciou a aplicação dos modelos de regressão para dados longitudinais, descritos a seguir. 


\section{Primeiro nivel de exposição}

Este primeiro nível é composto pela densidade populacional das unidades municipais e por indicadores que expressam a situação do desenvolvimento municipal, como condições de trabalho e renda e educação, operacionalizados pelo Índice Firjan de Desenvolvimento Municipal (IFDM) ${ }^{17}$ Foram agregados a este nível de determinação indicadores do Sistema Único de Saúde (SUS) presentes na Resolução da Comissão Intergestores Tripartite $n^{0} 5$, de 19 de junho de 2013, que trata dos indicadores municipais do rol de Diretrizes, Objetivos, Metas e Indicadores 2013-2015, a saber: (i) a proporção de internações por condições sensíveis à Atenção Básica, descritas na Portaria GM/ MS no 221, de 17 de abril de 2008; (ii) a cobertura de acompanhamento das condicionalidades de saúde do Programa Bolsa Família, estabelecidas pela Portaria Interministerial MS/MDS $\mathrm{n}^{0} 2.509$, de 18 de novembro de $2004 ;{ }^{15,16} \mathrm{e}$ (iii) a cobertura das equipes da Atenção Básica municipal, expressa em percentual da cobertura populacional alcançada pela Atenção Básica segundo o Contrato Organizativo da Ação Pública da Saúde. ${ }^{18}$

Segundo nível de exposição

Incorporou as condições ambientais específicas para variáveis climáticas: temperatura média anual, em percentis 10 e 90 das temperaturas no ano operacionalizadas em escala Celsius (unidade: ${ }^{\circ} \mathrm{C}$ ); precipitação pluviométrica acumulada anual, estimada em milímetros quadrados $\left(\mathrm{mm}^{2}\right)$; umidade relativa do ar, operacionalizada em percentis 10 e 90 das unidades; e altitude da sede municipal, em metros (m). Foram ainda considerados os índices de exposição costeira e cobertura vegetal, extraídos do Sistema de Vulnerabilidade Climática (SisVuClima). ${ }^{19}$

Terceiro nível de exposição

Considerou aspectos sociodemográficos locais: proporção populacional do sexo feminino; raça/cor da pele, expressa pelo percentual de indivíduos que se declaram brancos; e faixa etária, representada pelos percentuais de 0 a 14 ('Menor15'), 15 a 64 ('Adultos') e 65 anos ou mais anos ('Maior65'). Foram ainda acrescidas condições sociais de vulnerabilidade no nível municipal: expectativa de anos de estudo; proporção de cidadãos pobres; proporção da população residente em zona urbana; percentual de domicílios com água encanada; e percentual de domicílios com coleta de lixo.

Com o propósito de evitar efeitos de multicolinearidade, após análise da matriz de correlação, elaborou-se um modelo utilizando temperatura média anual e outro modelo considerando os percentis de 10 e 90.0 mesmo procedimento foi aplicado aos demais parâmetros climáticos de umidade e à faixa etária até 15 anos, adultos e maiores de 65 anos. Quanto à raça/cor da pele, a utilização das proporções de duas ou mais categorias implicaria multicolinearidade, de forma que se optou por considerar somente a proporção de brancos.

Para validar o uso do Google Maps pelo sistema de buscas, realizou-se uma verificação de concordância com o sistema Bing Maps a partir de uma amostra aleatória, proporcionalmente representativa dos municípios, composta por 1.123 endereços. Obteve-se uma medida de concordância para 0 índice Kappa ponderado por pesos quadráticos, de 0,42 ( $\mathrm{p}$-valor $<0,001$ ). Por fim, aplicou-se a técnica de análise multivariada com emprego da AF, estimando-se os componentes principais e a rotação ortogonal varimax das 30 doenças/agravos com maior volume de casos: arboviroses; doenças imunopreveníveis; hepatite B; causas externas; doenças negligenciadas: IST; hepatite C; zoonoses; e doenças ocupacionais.

0 projeto do estudo foi aprovado pelo Comitê de Ética em Pesquisa do Centro de Ciências da Saúde da Universidade Federal do Espírito Santo (CEP/CCS/ UFES): Parecer $n^{\circ} 2.991 .488$, emitido em 30 de outubro de 2018. Para garantir a confidencialidade no manuseio de dados de saúde, obteve-se da equipe a assinatura de termo de compromisso assegurando o sigilo dos dados trabalhados.

\section{Resultados}

Foram incluídos 347.789 registros de casos confirmados, de um total de 457.463 notificados. Os casos confirmados foram mais frequentes entre as mulheres $(51,1 \%)$, pardos $(31,7 \%)$, a faixa etária de $20-49$ anos (48,1\%) e residentes na região metropolitana de saúde $(60,3 \%) ; 46 \%$ dos casos investigados referiam-se a casos confirmados de dengue (46,3\%) (Tabela 1 ).

0 processo automatizado de extração das coordenadas geográficas apresentou sensibilidade de $74,4 \%$, obtendo localização de 258.910 coordenadas extraídas. Destas, 235.456 (90,9\%) mantiveram fidedignidade ao município correspondente. A sensibilidade observada apresentou uma pequena variação nos cinco anos de estudo, passando de $71,7 \%$, para 0 ano de 2011, a 79,3\% para 0 ano de 2012. A validação empregada utilizou oito combinações de 


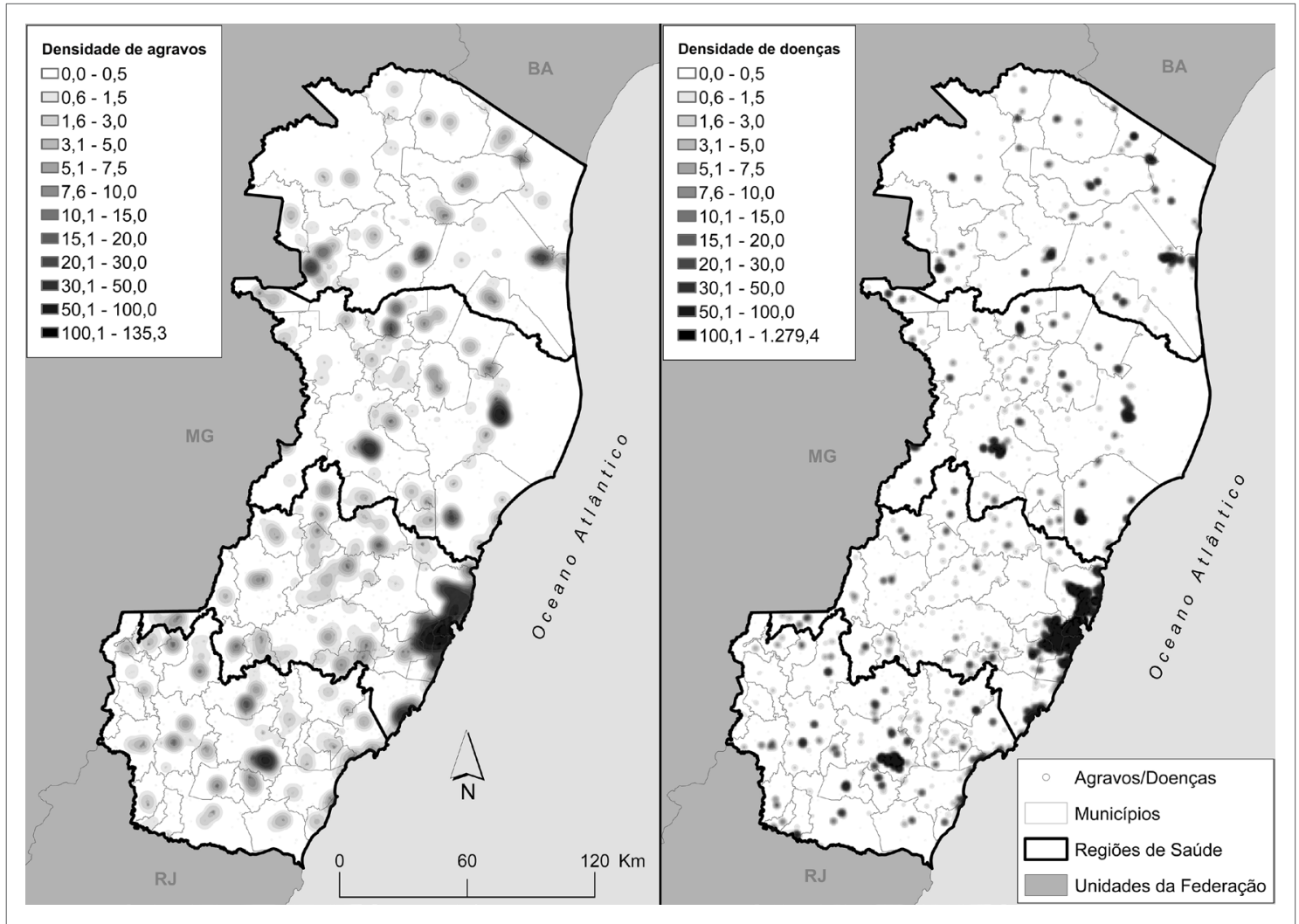

Figura 1 - Distribuição estimada das doenças e agravos de notificação no estado do Espírito Santo, 2011-2015

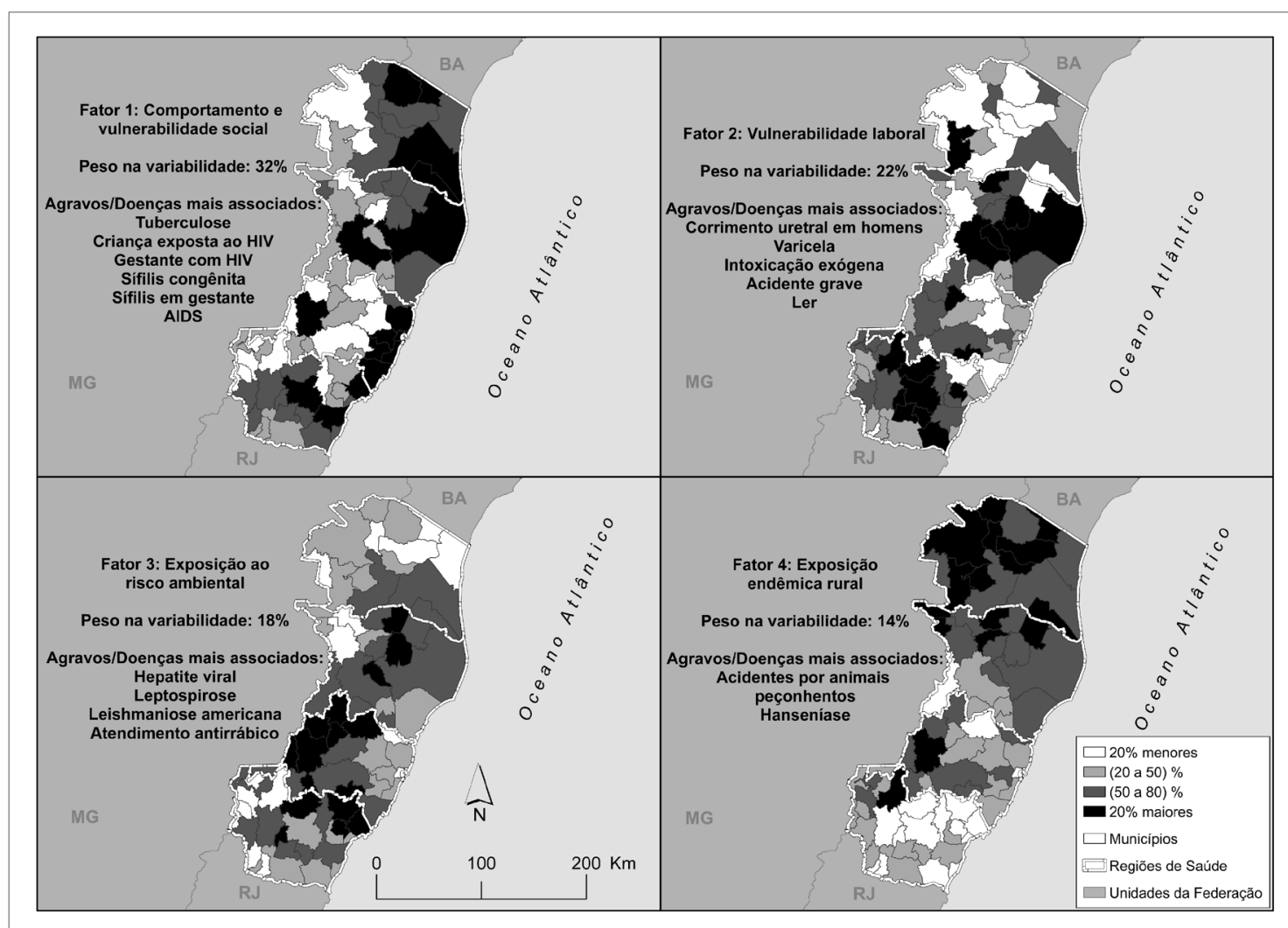

Figura 2 - Distribuição dos escores fatoriais para taxas de incidência das doenças e agravos de notificação no estado do Espírito Santo, 2011-2015 
Tabela 1 - Variáveis biológicas, geográficas e da categoria etiológica das doenças de notificação compulsória confirmadas em residentes no estado do Espírito Santo, 2011-2015

\begin{tabular}{|c|c|c|}
\hline Variáveis & N (347.789) & $\%$ \\
\hline \multicolumn{3}{|l|}{ Sexo } \\
\hline Feminino & 177.722 & 51,1 \\
\hline Masculino & 169.721 & 48,8 \\
\hline Indeterminado & 346 & 0,1 \\
\hline \multicolumn{3}{|l|}{ Raça/cor da pele } \\
\hline Branca & 91.913 & 26,4 \\
\hline Preta & 20.744 & 6,0 \\
\hline Amarela & 1.813 & 0,5 \\
\hline Parda & 110.364 & 31,7 \\
\hline Indígena & 781 & 0,2 \\
\hline Ignorada & 122.174 & 35,1 \\
\hline \multicolumn{3}{|l|}{ Faixa etária (anos) } \\
\hline$<1$ & 8.719 & 2,5 \\
\hline 01/abr. & 17.778 & 5,1 \\
\hline 05/set. & 22.446 & 6,5 \\
\hline out./19 & 56.996 & 16,4 \\
\hline $20-49$ & 167.439 & 48,1 \\
\hline $50-79$ & 60.724 & 17,5 \\
\hline$\geq 80$ & 3.141 & 0,9 \\
\hline Ignorada & 10.546 & 3,0 \\
\hline \multicolumn{3}{|l|}{ Região geográfica } \\
\hline Norte & 33.771 & 9,7 \\
\hline Central & 49.526 & 14,2 \\
\hline Metropolitana & 209.824 & 60,3 \\
\hline Sul & 54.668 & 15,7 \\
\hline \multicolumn{3}{|l|}{ Grupos etiológicos } \\
\hline Arbovirose & 161.176 & 46,3 \\
\hline Causas externas & 54.789 & 15,8 \\
\hline Doenças ocupacionais & 821 & 0,2 \\
\hline Doenças transmitidas por água e alimentos & 122 & 0,0 \\
\hline Hepatite sem confirmação & 177 & 0,1 \\
\hline Doenças imunopreveníveis & 21.010 & 6,0 \\
\hline Infecções sexualmente transmissíveis (IST) & 22.485 & 6,5 \\
\hline Doenças negligenciadas & 14.885 & 4,3 \\
\hline Zoonoses & 72.301 & 20,8 \\
\hline Residual (outras) & 23 & 0,0 \\
\hline
\end{tabular}


Tabela 2 - Modelo de regressão para condições de iniquidades e condições ambientais associadas aos agravos de notificação

\begin{tabular}{|c|c|c|c|c|c|c|c|c|c|}
\hline \multirow{2}{*}{$\begin{array}{l}\text { Modelo } \\
\text { hierarquizado }\end{array}$} & \multirow{2}{*}{ Variáveis } & \multicolumn{2}{|c|}{$1^{\circ}$ Etapa - Nível 1} & \multicolumn{2}{|c|}{$2^{\circ}$ Etapa - Nível 2} & \multicolumn{2}{|c|}{$3^{\circ}$ Etapa - Nível 3} & \multicolumn{2}{|c|}{$4^{\circ}$ Etapa - Modelo final } \\
\hline & & $\operatorname{Coef}(\beta)$ & p-valor & $\operatorname{Coef}(\beta)$ & p-valor & $\operatorname{Coef}(\beta)$ & p-valor & $\operatorname{Coef}(\beta)$ & p-valor \\
\hline \multirow{6}{*}{ Nível 1} & IntCdAtBca & 0,009 & $<0,001$ & 0,003 & 0,154 & 0,004 & 0,072 & 0,005 & 0,033 \\
\hline & CobCondSaud & 0,001 & 0,773 & & & & & & \\
\hline & CobAtencBsca & $-0,001$ & 0,707 & & & & & & \\
\hline & ifdm_edu & 0,034 & $<0,001$ & 0,019 & 0,003 & 0,010 & 0,100 & 0,015 & 0,013 \\
\hline & ifdm_emprend & $-0,003$ & 0,130 & & & & & & \\
\hline & Dens & $<0,001$ & 0,563 & & & & & & \\
\hline \multirow{10}{*}{ Nível 2} & Temp & & & - & - & & & & \\
\hline & Temp_p10 & & & 0,079 & 0,019 & 0,051 & 0,040 & 0,040 & 0,086 \\
\hline & Temp_p90 & & & $-0,041$ & 0,159 & & & & \\
\hline & $\log$ (Precip) & & & 0,009 & 0,904 & & & & \\
\hline & Umid & & & - & - & & & & \\
\hline & Umid_p10 & & & 0,006 & 0,376 & & & & \\
\hline & Umid_p90 & & & $-0,017$ & 0,196 & & & & \\
\hline & $\log (A \mid t)$ & & & 0,123 & 0,001 & 0,101 & 0,013 & 0,084 & 0,029 \\
\hline & Cobveg & & & 0,001 & 0,354 & & & & \\
\hline & ExpCosteira & & & 0,002 & 0,286 & & & & \\
\hline \multirow{10}{*}{ Nível 3} & Urb & & & & & - & 0,004 & $-0,010$ & 0,001 \\
\hline & Menor15 & & & & & - & - & & \\
\hline & Adultos & & & & & - & 0,842 & & \\
\hline & Maior65 & & & & & - & - & & \\
\hline & Branc & & & & & - & 0,744 & & \\
\hline & Mulh & & & & & 0,045 & 0,364 & & \\
\hline & ExpAnosEstud & & & & & - & 0,079 & -0.116 & 0,163 \\
\hline & AguEncan & & & & & 0,022 & 0,059 & 0,028 & 0,013 \\
\hline & ColetLixo & & & & & 0,016 & 0,441 & & \\
\hline & Pobr & & & & & $-0,014$ & 0,225 & & \\
\hline
\end{tabular}

Legenda:

IntCdAtBca: proporção de internações por condições sensíveis à Atenção Básica.

CobCondSaud: cobertura de acompanhamento das condicionalidades de saúde do Programa Bolsa Família.

CobAtencBsca: cobertura das equipes da Atenção Básica municipal expressa em percentual da cobertura populacional alcançada.

ifdm_edu: Indice Firjan de Desenvolvimento Municipal (IFDM) para educação.

ifdm_emprend:IFDM para condições de trabalho e renda.

Dens: densidade populacional.

Temp:temperatura média anual.

Temp_p10: percentil 10 da temperatura.

Temp_p90:percentil 90 da temperatura.

$\log$ (Precip):precipitação pluviométrica acumulada anual em escala logarítmica.

Umid: média anual da umidade relativa do ar.

Umid_p10: percentil 10 da umidade relativa do ar.

Umid_p90: percentil 90 da umidade relativa do ar.

$\log (\overline{A l t})$ :altitude da sede municipal em escala logarítmica.

Cobveg: índice de cobertura vegetal.

ExpCosteira: índice de exposição costeira.

Urb: proporção da população que reside em zona urbana.

Menor15: proporção da população com idade de 0 a 14 anos.

Adultos: proporção da população com idade de 15 a 64 anos.

Maior65: proporção da população com 65 anos ou mais.

Branc: percentual de indivíduos que se declaram brancos.

Mulh: percentual de indivíduos do sexo feminino.

ExpAnosEstud: expectativa de anos de estudo.

AguEncan: percentual de domicílios com água encanada.

ColetLixo: percentual de domicílios com coleta de lixo.

Pobr: proporção de pobres. 
Tabela 3 - Modelo de regressão para condições de iniquidades e condições ambientais associadas às doenças de notificação

\begin{tabular}{|c|c|c|c|c|c|c|c|c|c|}
\hline \multirow{2}{*}{$\begin{array}{l}\text { Modelo } \\
\text { hierarquizado }\end{array}$} & \multirow{2}{*}{ Variáveis } & \multicolumn{2}{|c|}{$1^{\circ}$ Etapa - Nível 1} & \multicolumn{2}{|c|}{$2^{\circ}$ Etapa - Nível 2} & \multicolumn{2}{|c|}{$3^{\circ}$ Etapa - Nível 3} & \multicolumn{2}{|c|}{$\begin{array}{l}4^{\circ} \text { Etapa - Modelo } \\
\text { final }\end{array}$} \\
\hline & & Coef $(\beta)$ & p-valor & Coef $(\beta)$ & p-valor & $\operatorname{Coef}(\beta)$ & p-valor & $\operatorname{Coef}(\beta)$ & p-valor \\
\hline \multirow{6}{*}{ Nível 1} & IntCdAtBca & 0,002 & 0,648 & & & & & & \\
\hline & CobCondSaud & $-0,003$ & 0,507 & & & & & & \\
\hline & CobAtencBsca & $-0,004$ & 0,186 & & & & & & \\
\hline & ifdm_edu & 0,015 & 0,190 & & & & & & \\
\hline & ifdm_emprend & 0,005 & 0,193 & & & & & & \\
\hline & Dens & 0,001 & $<0,001$ & 0,000 & 0,082 & $<0,001$ & 0,678 & $<0,001$ & 0,107 \\
\hline \multirow{10}{*}{ Nível 2} & Temp & & & 0,398 & $<0,001$ & 0,105 & 0,114 & 0,224 & $<0,001$ \\
\hline & Temp_p10 & & & - & - & & & & \\
\hline & Temp_p90 & & & - & - & & & & \\
\hline & $\log$ (Precip) & & & 0,624 & 0,083 & $-0,060$ & 0,812 & 0,155 & 0,549 \\
\hline & Umid & & & - & - & & & & \\
\hline & Umid_p10 & & & $-0,097$ & $<0,001$ & $-0,133$ & $<0,001$ & $-0,144$ & $<0,001$ \\
\hline & Umid_p90 & & & 0,246 & $<0,001$ & 0,317 & $<0,001$ & 0,326 & $<0,001$ \\
\hline & $\log (A \mid t)$ & & & 0,155 & 0,005 & 0,027 & 0,622 & 0,062 & 0,224 \\
\hline & Cobveg & & & $-0,001$ & 0,656 & & & & \\
\hline & ExpCosteira & & & $-0,007$ & $<0,001$ & 0,003 & 0,259 & 0,006 & 0,035 \\
\hline \multirow{10}{*}{ Nível 3} & Urb & & & & & 0,005 & 0,387 & & \\
\hline & Menor15 & & & & & - & - & & \\
\hline & Adultos & & & & & 0,221 & $<0,001$ & 0,165 & $<0,001$ \\
\hline & Maior65 & & & & & - & - & & \\
\hline & Branc & & & & & $-0,024$ & 0,001 & $-0,024$ & $<0,001$ \\
\hline & Mulh & & & & & 0,088 & 0,110 & & \\
\hline & ExpAnosEstud & & & & & 0,080 & 0,397 & & \\
\hline & AguEncan & & & & & 0,018 & 0,191 & & \\
\hline & ColetLixo & & & & & 0,022 & 0,309 & & \\
\hline & Pobr & & & & & 0,043 & 0,001 & 0,020 & 0,054 \\
\hline
\end{tabular}

Legenda:

IntCdAtBca: proporção de internações por condições sensiveis à Atenção Básica.

CobCondSaud: cobertura de acompanhamento das condicionalidades de saúde do Programa Bolsa Família.

CobAtencBsca: cobertura das equipes da Atenção Básica municipal expressa em percentual da cobertura populacional alcançada.

ifdm_edu: Indice Firjan de Desenvolvimento Municipal (IFDM) para educação.

ifdm_emprend:IFDM para condições de trabalho e renda.

Dens: densidade populacional.

Temp:temperatura média anual.

Temp_p10: percentil 10 da temperatura.

Temp_p90:percentil 90 da temperatura.

log(Precip):precipitação pluviométrica acumulada anual em escala logarítmica.

Umid:média anual da umidade relativa do ar.

Umid_p10: percentil 10 da umidade relativa do ar.

Umid_p90: percentil 90 da umidade relativa do ar.

$\log (\overline{A l t})$ :altitude da sede municipal em escala logarítmica.

Cobveg: índice de cobertura vegetal.

ExpCosteira: índice de exposição costeira.

Urb: proporção da populaçãão que reside em zona urbana.

Menor15: proporção da população com idade de 0 a 14 anos.

Adultos: proporção da população com idade de 15 a 64 anos.

Maior65: proporção da população com 65 anos ou mais.

Branc: percentual de indivíduos que se declaram brancos.

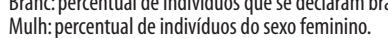

ExpAnosEstud: expectativa de anos de estudo.

AguEncan: percentual de domicílios com água encanada.

ColetLixo: percentual de domicílios com coleta de lixo.

Pobr: proporção de pobres. 
endereçamento. A combinação 'endereço completo', composta por logradouro, número, bairro, município, estado e país, alcançou maior sensibilidade, de 71,2\% para todo o período, variando entre $65,2 \%$, para 0 ano de 2012, a 75,3\% em 2011. Segundo a natureza da notificação, 82.054 (34,9\%) referiram-se à ocorrência de agravos e $153.402(65,2 \%)$ às doenças, cujas densidades de distribuição podem ser visualizadas na Figura 1: os agravos encontravam-se dispersos em áreas urbanas e rurais, enquanto as doenças se concentravam em áreas urbanas.

Quanto à análise comparativa entre os sistemas Google Maps e Bing Maps, destaca-se que 60\% das distâncias apresentaram-se entre 0 e 400 metros. Os valores apurados distribuíram-se em classes de distância sob uma curva bimodal, com a primeira entre 0 e 50 metros, representando $32,1 \%$ dos pontos georreferenciados. A segunda moda encontra-se entre 1 e $1,5 \mathrm{~km}$, representando $14,1 \%$ dos casos georreferenciados. Tais achados representam discordâncias sistemáticas entre as ferramentas de busca.

Os resultados obtidos para a análise de regressão das ocorrências dos agravos (Tabela 2) mantiveram, no nível 1 de exposição, as variáveis proporção de internações por condições sensíveis à Atenção Básica $(\beta=0,009$; p-valor $<0,001)$ e componente de educação do IFDM $(\beta=0,034$; $\mathrm{p}$-valor $<0,001)$. No segundo nível de exposição, as variáveis que apresentaram associação foram 0 percentil $10 \mathrm{da}$ temperatura $(\beta=0,079$; $\mathrm{p}$-valor $=0,019)$ e a altitude da sede do município em sua escala logarítmica $(\beta=0,123$; $p$-valor $=0,001)$. No terceiro nível, as variáveis do percentual de urbanização $(\beta=-0,013 ; p$-valor $=0,004)$, expectativa de anos de estudo $(\beta=-0,152 ; p$-valor $=0,079)$ e acesso a água encanada $(\beta=0,022 ; p$-valor $=0,059)$ estiveram associadas à incidência de agravos. 0 parâmetro de dependência temporal teve seu valor estimado ( $p o r \rho=0,843$; $p$-valor $<0,001$ ), indicando que o número de ocorrências de agravos em determinado ano esteve fortemente correlacionado à incidência do ano anterior.

De acordo com os resultados obtidos para a modelagem das ocorrências de doenças, $31 \%$ da variabilidade foram explicados pelo modelo (Tabela 3). Identificou-se associação positiva com a densidade populacional $(\beta=0,001 ; p$-valor $<0,001)$. No segundo nível de exposição, as variáveis de temperatura
( $\beta=0.398 ; p$-valor $<0,001)$, precipitação pluviométrica anual $(\beta=0,624 ; p$-valor $=0,083)$ e altitude $(\beta=0,155$; $\mathrm{p}$-valor $=0,005) \quad($ ambas em escala logarítmica $)$, percentil 10 da umidade relativa do ar $(\beta=-0,097$; $\mathrm{p}$-valor $<0,001)$, percentil $90 \mathrm{da}$ umidade relativa do ar $(\beta=0,246 ; p$-valor $<0,001)$ e índice de exposição costeira $(\beta=-0,007$; $p$-valor $<0,001)$ mantiveram-se associadas à incidência das doenças. No terceiro nível de exposição, foram encontradas associações entre as ocorrências e as variáveis indivíduos adultos $(\beta=0,221$; p-valor $<0,001)$, proporção de brancos $(\beta=-0,024$; p-valor $=0,001)$ e proporção de pobres $(\beta=0,043$; $p$ -valor=0.001). Após a inclusão de variáveis de um novo nível de exposição, alguns determinantes perderam significância no modelo final.

A técnica de $\mathrm{AF}$ foi aplicada às taxas de incidências das 30 doenças e agravos com maior número de casos entre todos os 78 municípios, sendo sua adequação verificada pelos testes de Bartllet ( $\mathrm{p}$-valor $<0,001)$ e estatística de Kaiser-Meyer-0lkin $(0,65)$. A AF resultou em quatro fatores, que representaram $86 \%$ da variabilidade dos dados. Esses fatores foram tipificados: Fator 1, Comportamento e vulnerabilidade social; Fator 2, Vulnerabilidade laboral; Fator 3, Exposição ao risco ambiental; e Fator 4, Exposição endêmica rural. Assim, foi possível agrupar os municípios em quatro categorias, representadas na Figura 2.

\section{Discussão}

Observou-se associação entre os agravos de notificação compulsória e as condições de internações sensíveis à Atenção Básica, o índice de desenvolvimento municipal de educação, a temperatura, a umidade relativa do ar, a altitude e a taxa de urbanização municipal, a expectativa de anos de estudo e a oferta de água encanada. A ocorrência das doenças esteve associada com variáveis de densidade populacional, temperatura, precipitação pluviométrica, umidade relativa do ar, altitude e índice de exposição costeira, além das proporções de adultos, de brancos e de pobres na população, sugerindo que a associação com as condições socioambientais e iniquidades corroboram a incidência das DANC. ${ }^{21}$

Uma limitação do estudo diz respeito ao baixo poder explicativo para o modelo dos agravos. Trata-se de ocorrências/notificações de naturezas bastante distintas, como acidentes de trabalho grave, eventos adversos pós-vacinação e acidentes provocados por animais peçonhentos, sendo necessário um detalhamento de 
risco específico para cada uma dessas condições no nível proximal. Já para o modelo aplicado a doenças, obteve-se maior coeficiente de determinação, posto que as condições de saúde da população de uma região guardam relações entre o território e a doença, de forma inequívoca, intrínseca e socialmente determinada.

Os impactos das mudanças climáticas podem se refletir em diversos cenários, como migrações em massa e relações econômicas, acometendo segmentos populacionais que, por sua vez, sobrecarregam os sistemas de saúde. 0 conhecimento desses impactos sobre as DANC pode contribuir para sua mitigação e possibilitar ao poder público lidar com as populações afetadas. ${ }^{22-24}$

Barcellos et al..$^{25}$ apontam que as experiências municipais na abordagem de dados em saúde necessitam de melhorias na qualidade dos dados, para emprego de técnicas de geoprocessamento. Destaca-se que os relatos das experiências se limitavam a abrangências municipais, chamando a atenção para a originalidade do presente trabalho em sua abordagem estadual do território do Espírito Santo. Outros achados, como o de Bando et al., ${ }^{26}$ apontam que o desemprego no Brasil está correlacionado positivamente com o homicídio, e que o suicídio estaria relacionado com alto nível socioeconômico. Análises apontando para possíveis condições de generalização motivaram as estimativas da $\mathrm{AF}$, permitindo identificar fatores comuns aos grupos etiológicos, favorecendo interpretações generalizadas de suas ocorrências. Destacam-se (i) o 'Comportamento e vulnerabilidade social', representado por agravos de infecções sexualmente transmissíveis, que teve sua localização na faixa litorânea, além da tuberculose nos municípios populosos do interior do estado; (ii) a 'Vulnerabilidade laboral', referente às doenças relacionadas ao trabalho, associada às atividades de risco potencial no ambiente de trabalho; (iii) a 'Exposição ao risco ambiental', em virtude da presença de agentes bióticos, físicos e geográficos em sua composição, caracterizada pelas condições intrinsecamente associadas ao ambiente rural; e finalmente, (iv) a 'Exposição endêmica rural', representada por agravos intimamente ligados ao meio rural, representados pelos acidentes com animais peçonhentos e a hanseníase, que apresentou elevadas taxas de incidência nos municípios do noroeste do Espírito Santo. Também cumpre mencionar a região da divisa com o estado de Minas Gerais, onde se identifica, na sub-região do Caparaó, composta por 11 pequenos municípios do sudoeste do Espírito Santo, próximos a Minas Gerais, a presença de cluster para ocorrência de doenças tipificadas pelos fatores de 'Comportamento e vulnerabilidade social' e 'Exposição ao risco ambiental'. Analogamente, na região Norte do estado, identifica-se outro cluster para as condições tipificadas como 'Vulnerabilidade laboral' e, com um peso menor, para 'Exposição endêmica rural'.

Segundo Freiler, ${ }^{27}$ no estudo dos bairros "pobres" da cidade de Toronto, em 2004, as concentrações de pobreza e condições ambientais desfavoráveis em relação ao bem-estar dos indivíduos deparam-se com o emprego das redes sociais como um bloco de construção, em busca de soluções próprias para problemas locais, inclusive os de saúde. Nesse sentido, pretende-se que a metodologia desenvolvida neste trabalho seja replicada nos grupos etiológicos específicos em escala municipal, contribuindo para a formulação das políticas públicas de saúde.

A ferramenta de extração das coordenadas geográficas sugere que o desenvolvimento de sistemas próprios no nível local pode contribuir com melhorias na validação do banco de dados da morbidade compulsória. Acrescentam-se evidências sobre condições ambientais explicativas de maior amplitude sobre possíveis relações causais do processo saúde-doença associado às DANC. A moderada concordância encontrada sinaliza para a fragilidade quanto ao uso, entre os diversos componentes dos endereços dos sistemas de informações em saúde. Tal situação é conhecida no âmbito da Saúde Pública, face à qualidade do preenchimento dos sistemas de informações e a precariedade da base de endereçamentos disponíveis nas cidades brasileiras. ${ }^{28,29}$ Contudo, os fenômenos não são diretamente observáveis por diferentes limitações, a exemplo da subnotificação, dificuldades no acesso aos serviços de saúde, lacunas existentes nos processos subsequentes à notificação, confirmação laboratorial e descarte de casos. Assim, torna-se relevante o uso de técnicas multivariadas, que contribuam para 0 entendimento das inter-relações das condições ambientais e sociais voltadas à determinação das incidências no caso das DANC. Técnicas multivariadas favorecem a compreensão do território, as condições de vida existentes, a ressignificação das complexidades dos processos adaptativos, confirmando que o campo da Saúde, isoladamente, não consegue assegurar uma qualidade 
de vida e saúde digna à população. Conforme sugerem os resultados deste trabalho, as condições climático-ambientais e as iniquidades sociais estão associadas à ocorrência das DANC no Espírito Santo.

Conclui-se que houve associação dos agravos de notificação com as condições ambientais de temperatura, precipitação pluviométrica, umidade relativa do ar, altitude da sede municipal; e com os indicadores sociais de internações por condições sensíveis à Atenção Básica, índice de desenvolvimento municipal de educação, proporção de residentes em zona urbana, anos de estudo e percentual de domicílios atendidos pela rede de água encanada. As doenças estiveram associadas a densidade populacional, indicadores climático-ambientais de temperatura, precipitação pluviométrica, umidade relativa do ar, altitude da sede municipal e índice de exposição costeira; e aos grupos sociodemográficos de raça/cor da pele branca, faixa etária de 15 a 65 anos e proporção de pobres.

\section{Referências}

1. Henrique, FC. Ares, águas e lugares. In: Textos hipocráticos: o doente, o médico e a doença. Rio de Janeiro: Fiocruz: 2005. p. 91-129.

2. Ribeiro SK, Santos AS, editores. Mudanças climáticas e cidades: relatório especial do painel brasileiro de mudanças climáticas. Rio de Janeiro: PBMC; 2016. $166 \mathrm{p}$.

3. Ministério da Saúde (BR). Saúde ambiental: guia básico para construção de indicadores. Brasília, DF: MS; 2011. 124 p.

4. Medronho RA, Perez M. A distribuição das doenças no espaço e no tempo. In: Medronho RA, Carvalho DM, Bloch KV, Luiz RR, Werneck G. Epidemiologia. São Paulo: Atheneu; 2002. p. 57-71.

5. Minayo MCS, Miranda AC, organizadoras. Saúde e ambiente sustentável: estreitando-nos [e-book]. Rio de Janeiro: Editora FIOCRUZ: 2002. 344 p. doi: https://doi.org/10.7476/9788575413661.

6. Teixeira MG, Penna GO, Risi JB, Penna ML, Alvim MF, Moraes JC, et al. Seleção das doenças de notificação compulsória: critérios e recomendações para as três esferas de governo. Informe Epidemiol SUS. 1998:7(1): 7-28. http://dx.doi.org/10.5123/S010416731998000100002 .
Tais achados identificam os determinantes espaciais envolvidos na distribuição das DANC no estado do Espírito Santo.

\section{Contribuição dos autores}

Andrade RLM, Bertolde AI e Dantas A contribuíram na concepção e delineamento do artigo, análise e interpretação dos dados e redação da primeira versão do manuscrito. Spala MR, Silva G e Ribeiro FAS contribuíram na análise e interpretação dos dados, redação e revisão crítica do conteúdo do manuscrito. Silva RC, Morellato SA e Ramalho WM contribuíram na concepção do artigo, análise e interpretação dos resultados, redação e revisão crítica do conteúdo do manuscrito. Todos os autores aprovaram a versão final do manuscrito e são responsáveis por todos os seus aspectos, incluindo a garantia de sua precisão e integridade.

7. Hino P, Villa TCS, Cunha TN, Santos CB. Distribuição espacial de doenças endêmicas no município de Ribeirão Preto (SP). Cienc Saude Colet. 2011:16(supl. 1): 1289-94. doi: http://dx.doi. org/10.1590/\$1413-81232011000700062.

8. Carvalho MS, Souza-Santos R. Análise de dados espaciais em saúde pública: métodos, problemas, perspectivas. Cad Saude Publica. 2005;21(2):36178. doi: https://doi.org/10.1590/S0102311X2005000200003.

9. Soares KKS, Prado TN, Zandonade E, MoreiraSilva SF, Miranda AE. Análise espacial da sífilis em gestantes e sífilis congênita no estado do Espírito Santo, 2011-2018. Epidemiol Serv Saude. 2020;29(1):e2018193. doi: https://doi.org/10.5123/ s1679-49742020000100018.

10. Bastos MJRP, Pereira JA, Smarzaro DC, Costa EF, Bossanel RCL, Oliosa DMS, et al. Análise ecológica dos acidentes e da violência letal em Vitória, ES. Rev Saude Publica. 2009;43(1):123-32. doi: https://doi. org/10.1590/S0034-89102009000100016.

11. Brasil. Lei n. 6.259 de 30 de outubro de 1975. Portaria Ministério da Saúde. GM 204/2016. Dispõe sobre as conceituações para agravo e doença. 2016 [citado 10 fev 2021]. Disponível em: http://www. planalto.gov.br/ccIVIL_03/leis/L6259.htm 
12. Presidência da República (BR). Decreto n. 78.231, de 12 de agosto de 1976. Regulamenta a Lei $\mathrm{n}^{\circ}$ 6.259, de 30 de outubro de 1975, que dispõe sobre a organização das ações de Vigilância Epidemiológica, sobre o Programa Nacional de Imunizações, estabelece normas relativas à notificação compulsória de doenças, e dá outras providências. Brasília, DF: Diário Oficial da União; 13 ago. 1976 [citado 10 fev 2021]. Disponível em: http://www.planalto.gov.br/ ccivil_03/decreto/1970-1979/D78231.htm

13. Ministério da Saúde (BR). Portaria de consolidação n. ${ }^{\circ} 4$ de 28 de setembro de 2017. Dispõe sobre consolidação das normas sobre os sistemas e os subsistemas do Sistema Único de Saúde. Brasília, DF: Diário Oficial da União; 2017 [citado $10 \mathrm{fev}$ 2021]. Disponível em: http://bvsms.saude.gov.br/bvs/ saudelegis/gm/2017/prc0004_03_10_2017.html

14. Fitz PR. Geoprocessamento sem complicação. São Paulo: Oficina de Textos; 2008.

15. Orgalização Mundial da Saúde. CID-10: classificação estatística internacional de doenças e problemas relacionados à saúde. São Paulo: USP; 1997.

16. Prado DGO. Ajuste de cópulas bivariadas via marginal na diagonal e teste para dependência de valores extremos via ajuste de função de Pickands por polinômios de Bernstein [tese]. [Lavras]: Universidade Federal de Lavras; 2016. 126 p.

17. Federação das Indústrias do Estado do Rio de Janeiro. Índice FIRJAN de Desenvolvimento Municipal - 2014. Rio de Janeiro: FIRJAN; 2010 [citado 10 fev 2021]. Disponível em: https://www.firjan.com.br/data/ files/E8/06/F0/D5/58E1B610E6543AA6A8A809C2/ Metodologia\%20IFDM\%20-\%20Final.pdf

18. Ministério da Saúde (BR). Caderno de diretrizes, objetivos, metas e indicadores: 2013-2015. 2a ed. Brasília, DF: MS; 2014 [citado 10 fev 2021]. Disponível em: http://bvsms.saude. gov.br/bvs/publicacoes/caderno_diretrizes_ objetivos_2013_2015_2edicao.pdf

19. Sistema de Vulnerabilidade Climática - Projeto: construção de indicadores de vulnerabilidade da população como insumo para a elaboração das ações de adaptação à mudança do clima no Brasil [Internet]. [Brasília, DF: Ministério do Meio Ambiente; 2017]. Versão 1.03 - Dez/2017 [citado 10 fev 2021]. Disponível em: http://www.sisvuclima. com.br/
20. Instituto Brasileiro de Geografia e Estatística. Resultado dos dados preliminares do censo: 2000. [Brasília, DF]: 2000.

21. Secretaria da Saúde do Espírito Santo. Plano diretor de regionalização: PDR, ES 2011. [Vitória, ES: SESA; 2011]. Disponível em: PDR_ PlanoDiretordeRegionalizacao_ES_2011.pdf

22. Ministério da Saúde (BR). Mudanças climáticas e ambientais e seus efeitos na saúde: cenários e incertezas para o Brasil. Brasília, DF: OPAS; 2008. 40 p.

23. Sousa TCM, Amancio F, Hacon SS, Barcellos C. Doenças sensíveis ao clima no Brasil e no mundo: revisão sistemática. Rev Panam Salud Publica. 2018;42:e85. doi: http://doi.org/10.2663/ RPSP.2018.85.

24. Nahas MIP. Construção de modelo conceitual para o Índice de Vulnerabilidade Geral: etapa 1. In: FIOCRUZ. Construção de indicadores de vulnerabilidade da população como insumo para a elaboração das ações de adaptação à mudança do clima no Brasil. Belo Horizonte: Centro de Pesquisas René Rachou, FIOCRUZ; 2015. 82 p. Relatório.

25. Barcellos C, Ramalho WM, Gracie R, Magalhães MAFM, Fontes MP, Skaba D. Georreferenciamento de dados de saúde na escala submunicipal: algumas experiências no Brasil. Epidemiol Serv Saude. 2008:17(1):59-70. doi: http://dx.doi.org/10.5123/ S1679-49742008000100006.

26. Bando DH, Lester D. An ecological study on suicide and homicide in Brazil. Cienc Saude Colet. 2014;19(4):1179-89. doi: https://doi. org/10.1590/1413-81232014194.00472013.

27. Freiler $\mathrm{C}$. Why strong neighborhoods matter: implications for policy and practice [Internet]. Toranto: Strong Neighborhoods Task Force. Nov 2004 [citado 10 fev 2021]. Disponível em: http:// www.urbancentre.utoronto.ca/pdfs/curp/SNTF_WhyNeighbourhoods-Mat.pdf

28. Sousa TC; Amancio F; Hacon SS; Barcellos C. Doenças sensíveis ao clima no Brasil e no mundo: revisão sistemática. Revista Panamericana de Salud Pública, Jul 2018, Volume 42.

29. [Ministério da Saúde (BR)]. Roteiro para uso do Sinan NET: análise da qualidade da base de dados e cálculo de indicadores epidemiológicos e operacionais. [Brasília, DF: MS]; 2008. (Caderno Geral). (http://dtr2004.saude.gov.br/SINANweb/novo/ Documentos/SINANNET/analise/GERAL.pdf) 
30. [Ministério da Saúde (BR)]. Roteiro para Uso do Sinan NET: análise da qualidade da base de dados e cálculo de indicadores epidemiológicos e operacionais [Brasília, DF: MS]; 2008. (Acidentes

\section{Abstract}

Objective: To analyze association between climaticenvironmental conditions and occurrence of compulsorily notifiable diseases and health problems, in the state of Espirito Santo, Brazil, 2011-2015. Methods: This was an ecological study of municipality clusters calculated based on cases confirmed on the Notifiable Health Conditions Information System for the period 2011-2015. Results: Notifications were more frequent among females (51.1\%); people of brown race/skin color (31.7\%); in the 20 -49 year age group (48.1\%) and in the Metropolitan Health Region (60.3\%). The factors associated with health problems were ambulatory care sensitive conditions $(p$-value $<0.001)$; education development index $(p$-value $<0.001)$; temperature $(p$-value $=0.019)$ and degree of urbanization ( $p$-value $=0.004)$. Diseases were associated with population density ( $p$-value $<0.001)$; temperature ( $p$-value $<0.001)$, humidity ( $p$-value $<0.001)$ and altitude ( $p$-value $=0.005)$. Conclusion: Health problems were positively associated with ambulatory care sensitive conditions, the education development index and temperature; but negatively associated with degree of urbanization. Diseases were positively associated with the factors mentioned.

Keywords: Incidence; Disease Notification; Socioeconomic Factors; Spatial Analysis; Environment. por Animais Peçonhentos). http://dtr2004.saude.gov. br/SINANweb/novo/Documentos/SINANNET/analise/ animPeconhentos.pdf

\section{Resumen}

Objetivo: Analizar la asociación de las condiciones climáticas-ambientales y la ocurrencia de enfermedades y trastornos de notificación obligatoria en el estado de Espírito Santo, Brasil. Métodos: Estudio ecológico de los agregados municipales, a partir de casos confirmados en el Sistema de Información de Agravamientos de Notificación, período 2011-2015. Resultados: Las notificaciones fueron más frecuentes en el sexo femenino (51,1\%); raza/ color de piel parda (31,7\%); de 20-49 años (48,1\%) y región metropolitana de salud (60,3\%). Los factores asociados a los agravamientos fueron sensibles a la atención primaria $(p<0,001)$; los índices de desarrollo educativo $(p<0,001)$; la temperatura $(p=0,019)$ y el grado de urbanización $(p=0,004)$. Los agravamientos estuvieron asociados con la densidad de población $(p<0,001)$; la temperatura $(p<0,001)$, la humedad $(p<0,001)$ y la altitud $(p=0,005)$. Conclusión: Los agravamientos se asociaron positivamente con las condiciones sensibles a la atención primaria, con el indice de desarrollo educativo y la temperatura; y negativamente con el grado de urbanización. Las enfermedades se asociaron positivamente con los factores mencionados.

Palabras clave: Incidencia; Notificación de Enfermedades; Factores Socioeconómicos; Análisis Espacial; Ambiente.

Recebido em 25/06/2020

Aprovado em 06/01/2021 